\title{
Miranda
}

Revue pluridisciplinaire du monde anglophone /

Multidisciplinary peer-reviewed journal on the English-

speaking world

16 | 2018

L'expérimental dans la littérature et les arts

contemporains

\section{War Requiem, Opéra de Lyon (octobre 2017) : quand sobriété et puissance se mêlent}

\section{Muriel Joubert}

\section{OpenEdition}

\section{Journals}

Édition électronique

URL : http://journals.openedition.org/miranda/11802

DOI : 10.4000/miranda.11802

ISSN : 2108-6559

\section{Éditeur}

Université Toulouse - Jean Jaurès

Référence électronique

Muriel Joubert, « War Requiem, Opéra de Lyon (octobre 2017) : quand sobriété et puissance se mêlent », Miranda [En ligne], 16 | 2018, mis en ligne le 06 juin 2018, consulté le 16 février 2021. URL http://journals.openedition.org/miranda/11802 ; DOI : https://doi.org/10.4000/miranda.11802

Ce document a été généré automatiquement le 16 février 2021.

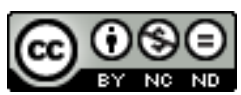

Miranda is licensed under a Creative Commons Attribution-NonCommercial-NoDerivatives 4.0 International License. 


\title{
War Requiem, Opéra de Lyon (octobre 2017) : quand sobriété et puissance se mêlent
}

\author{
Muriel Joubert
}

1 Benjamin Britten a composé son War Requiem pour l'inauguration, en mai 1962, de la Cathédrale de Coventry, détruite pendant la Seconde Guerre mondiale. Ce requiem « de guerre » prend une place particulière à la fois pour Britten et dans l'Histoire de la musique. D'une part, le War Requiem, conçu en pleine guerre froide, constitue en quelque sorte un aboutissement pour le compositeur antimilitariste de 49 ans qui pense à écrire depuis de nombreuses années une œuvre destinée à la réconciliation des peuples. La commande pour la cathédrale de Coventry n'est ainsi que le catalyseur d'un projet sous-jacent, annoncé par certaines œuvres : Ballad of Heroes en 1939 dédié à des combattants anglais de la guerre civile espagnole ; Sinfonia da Requiem en 1940 ; Canticle II : Abraham and Isaac en 1952, pour ne citer que quelques exemples.

Britten choisit alors d'enrichir le texte liturgique traditionnel en latin de poèmes choisis du poète de guerre anglais Wilfred Owen, engagé volontaire en tant que sousofficier lors de la Première Guerre mondiale, et qui y laissa sa vie sept jours avant l'armistice, à l'âge de 25 ans. Ces poèmes en langue anglaise métamorphosent le genre du requiem qui perd alors de sa fonction liturgique pour revêtir une dimension plus dramaturgique. Les poèmes sont chantés par des solistes (ténor, baryton) et évoquent des images de guerre, la " colère des canons ", l'abattement des soldats, leur peur, leur rencontre de la Mort et l'« odeur verte de son haleine", l'impuissance du soleil et finalement celle de la parole religieuse, à travers la métabole détournée du sacrifice d'Isaac. La guerre y apparait comme une absurdité inégalée : " Je suis l'ennemi que tu as tué, mon ami. [...] Dormons maintenant... ». Les deux ennemis sont le ténor (dont Britten voulait qu'il soit anglais : Peter Pears dans la version de création) et le baryton (allemand: Dieter Fischer-Dieskau), alors qu'une chanteuse de nationalité russe emprunte des extraits de la liturgie latine pour figurer dans un rôle qui oscille entre la prédication et la colère. Autour de ces solistes gravitent deux chœurs - le chœur mixte 
et le chœur de garçons, tous deux représentant la parole religieuse - ainsi que deux orchestres: le grand orchestre pour accompagner le chœur mixte et l'orchestre de chambre pour les deux solistes chantant les poèmes d'Owen. Trois mondes différents semblent se positionner dans l'espace symbolique comme scénique: le monde divin (chœur mixte et orchestre, ainsi que la soprano), celui de l'innocence et de l'idéal rêvé (le chœur des garçons accompagné par un orgue) et enfin celui des humains, des soldats englués dans la vie réelle (le ténor et le baryton accompagnés par l'orchestre de chambre).

Le War Requiem rassemble tous les ingrédients d'une dramaturgie. En 1989, Derek Jerman l'avait montré en décidant de réaliser un film qui avait pour unique bande sonore l'œuvre de Britten/Owen. L'Opéra de Lyon en proposait la première version scénique en octobre 2017, confiant la mise en scène à Yoshi Oïda, un habitué des opéras de Britten : Curlew River à Aix-en-Provence en 1998, alors qu'il avait 65 ans, puis Death in Venice et Peter Grimes à Lyon même (respectivement en 2009 et en 2014).

4 « Je me sens un peu coupable, nous confiait Yoshi Oïda peu avant la pré-générale ${ }^{1}$, car Britten a écrit cette œuvre dans un contexte symphonique, et pas pour l'opéra. Sa musique est très belle! Ce n'est pas la peine de rajouter du visuel ! [...] Je n'ai pas souhaité ajouter quoi que ce soit à la musique ». De même, Derek Jerman n'avait pas introduit un seul bruitage, mais c'était sans doute pour respecter la condition incontournable de la maison de production Decca Records, cette dernière lui ayant accordé le droit d'utiliser l'enregistrement de 1963 réalisé par Britten lui-même (avec Peter Pears, Fischer-Dieskau et la Russe Galina Vichnevkaia, alors épouse de Mstislav Rostropovitch). L'œuvre pacifiste qu'est le War Requiem étant transposable à toutes les époques, et notamment à la nôtre, il aurait été tentant de rajouter des dialogues réactualisant le contexte. Mais Yoshi Oïda, dans son souci de respecter l'œuvre de Britten, n'en a rien fait. Il dit avoir été très touché par les poèmes de Wilfred Owen, où subsistent beaucoup de doutes encore d'actualité : « la vie, la mort, la religion, la guerre, l'humain... ».

Ne voulant surtout pas " théâtraliser » le War Requiem, c'est-à-dire refusant de faire de cette œuvre un spectacle grandiloquent, Yoshi Oïda a réalisé une succession de tableaux dont l'enchaînement dramaturgique est irréprochable. Le premier montre un mur sombre sur lequel se détachent des hommes et des femmes (le chœur) revêtus de cirés colorés et portant des parapluies (costumes de Thibault Vancraenenbrœck). Devant eux, une rangée de morts enveloppés chacun dans un linceul. Le chœur chrétien porteur de la parole liturgique de Britten revêt ici la figure du peuple, comme touché par la mort des leurs et assistant à leurs funérailles un jour de pluie.

6 La mort, omniprésente dans le texte d'Owen, l'est aussi dans la mise en scène de Yoshi Oïda, qui dit d'ailleurs s'être intéressé à la sensation qu'a pu éprouver le poète lors de l'écriture de ces vers, dans des conditions effroyables. En outre, l'idée d'introduire dans sa poésie son expérience douloureuse de la guerre lui avait été suggérée par son ami le poète Siegfried Sassoon, rencontré à l'hôpital où il soignait une neurasthénie due au traumatisme de la guerre, ainsi par que son propre médecin. Sur la scène de l'Opéra de Lyon, les cadavres sont ainsi omniprésents, sous différentes formes : corps enveloppés, seuls habits posés sur le sol, tête de mort, poupées et marionnettes... L'ironie dont fait preuve Wilfred Owen dans le premier duo du ténor et du baryton ("Out There, we'we walked quite friendly up to Death»), soulignée par la marche funèbre, les cymbales, les violons ou le piccolo, n'échappe pas au metteur en scène: les deux protagonistes s'y 
disputent une tête de mort. "Oh non, jamais la Mort ne fut notre ennemie ! Nous avons ri d'elle, avons fait cause commune, veille branche!», entend-on à travers les vers d'Owen.

7 Certes, Yoshi Oïda ne souhaite pas sur-théâtraliser cette œuvre jouée à l'origine dans une église, mais la scène d'opéra l'oblige à en exacerber la dramaturgie intérieure. Premièrement, Yoshi Oïda choisit de personnifier les chanteurs, comme l'avait fait indirectement Derek Jerman dans son adaptation filmique (on ne voit pas de chanteurs dans le film, l'ensemble de la musique étant extra-diégétique). Naturellement, le drapeau anglais identifie le ténor, soldat anglais, incarné à Lyon par le chanteur américain Paul Groves ; le drapeau allemand distingue le baryton, soldat allemand représenté ici par l'estonien Lauri Vasar. Quant à la soprano incarnée ici par la russe Ekaterina Scherbachenko, son rôle est ambigu chez Britten: chantant la parole liturgique, elle s'impose dans un ton de prescription implacable (" Le livre achevé sera lu, où tout se trouve consigné pour ouvrir le procès du monde ", entend-on dans le Liber scriptus), ne laissant alors aucune chance à la salvation humaine. Et pourtant, sa colère semble paradoxalement dénoncer les absurdités contraires, celles du monde des hommes et du monde divin (Rex remendae), jusqu'à être emplie d'une tristesse irrémédiable (Lacrimosa). Selon le souhait de Britten, la soprano aurait dû chanter le texte biblique en langue anglaise, comme se situant entre le monde du Dieu et celui des soldats et des humains, mais le compositeur en aurait abandonné l'idée car la chanteuse russe maîtrisait mal l'accent anglais (Vincent-Arnaud, 2005 : 201-217). Yoshi Oïda choisit de personnifier complètement le rôle de la soprano : "son mari ou son amant était soldat, et il est mort. Toute l'histoire, c'est son histoire à elle. Elle est le centre de cet opéra, et doit chanter de manière dramatique ». Derek Jerman en avait fait la bien-aimée de Owen ; Oïda étend le rôle à la personnification de la veuve de guerre. Les portraits posés sur la scène à la toute fin du spectacle sont autant d'hommes pleurés par leurs bien-aimées, celui de Wilfred Owen trônant parmi eux.

D'autre part, Yoshi Oïda choisit de mettre sur scène certains objets forts des poèmes d'Owen, tel ce canon-bras (« Lève-toi lentement, ô long bras noir, Grand canon braqué vers le ciel, prêt à maudire») manipulé par le baryton dans le Dies Irae, comme accompagnant la sentence soulignée par les cuivres. Malgré un feu jaillissant brièvement dans le Dies Irae, Yoshi Oïda garde l'expressivité maximale pour le Libera me, respectant le climax graduel proposé par le compositeur : « Britten change subtilement de couleur dans son opéra, au fur et à mesure, selon les personnes et les poèmes, les ambiances. Il amène peu à peu le climax vers la fin, avec le Libera me et le duo "Strange meeting" entre le Ténor et le Baryton. J'ai simplement voulu suivre le cheminement de Britten. » Le Libera me fait exploser toute la tension précédemment retenue, à travers des images vidéos projetées par vignettes sur le mur : une polyphonie de films supposés d'époque se superpose, montrant des marées de bataillon, des soldats marchant dans la boue, des scènes de batailles en mer, le champignon d'Hiroshima qui marqua tant Yoshi Oïda, alors au Japon, pendant la Seconde Guerre mondiale. Devant le mur d'images vidéo, la veuve-soprano lave le linge des soldats dans un bac dont l'eau va se transformer en sang. Puis le mur finit par tomber en ruines, pierre par pierre, comme symbolisant les multiples murs du monde à travers lesquels se sont cristallisées les tensions de l'Histoire - les murailles de Jéricho, le mur des Lamentations à Jérusalem, les murs de Berlin, de Belfast, la muraille de Chine, mais aussi les ruines de la cathédrale de Coventry qui jouxtent l'édifice reconstruit, ou encore celles de Palmyre. Le décor, pensé et réalisé par Tom Schenk, également partenaire de Yoshi Oïda dans 
Death in Venice et Peter Grimes, est à la fois sobre et puissant dans sa capacité à revêtir des expressions d'intensités variables, modulables selon les couleurs des costumes et des lumières (conçues par Lutz Deppe).

9 Néanmoins, Yoshi Oïda s'est interrogé sur la mise en scène à l'opéra d'une œuvre a priori chrétienne : le public n'étant pas forcément chrétien, comment atteindre tout le monde ? D'où son choix d'une forme de mise en abyme de la narration du War Requiem. Une classe d'écoliers, placée côté cour de la scène, regarde le spectacle du War Requiem, celui, plus exactement, de la guerre. « Les chanteurs et le chœur montrent le spectacle de la Première Guerre mondiale aux enfants ", dit Oïda. Le spectacle trouve son paroxysme lors de la scène du Sacrifice d'Isaac, où des marionnettes sont actionnées par les deux solistes, devenus clairement deux enseignants d'histoire. Moins ostentatoire que la scène de sacrifice de Derek Jerman, où Isaac n'était autre que Wilfred Owen se faisant trancher la gorge sur la table de sacrifice, la scène n'en reste pas moins dramatique avec les marionnettes de Yoshi Oïda, puisque le poète avait choisi dans sa relecture de laisser Abraham sacrifier son fils (" Mais le vieil homme ne l'entendit pas ainsi, et tua son fils »). Les enfants du chœur assistent à cette scène insoutenable, à peine atténuée par quelques effets comiques liés à la manipulation des marionnettes, puis vont prendre part à la scène de spectacle, comme vérifiant la véracité de l'Histoire, celle de la mort de chacun des enfants (" Mais le vieil homme ne l'entendit pas ainsi, et tua son fils et la moitié des enfants d'Europe, un par un »), avant d'entonner une prière: "Nous t'offrons, Seigneur, ces hosties, ces prières de louange... ». Ainsi, selon le vœu du metteur en scène, la mise en abyme agit comme un écran supplémentaire : le public regarde la scène qui se déroule - mélange de discours chrétien et de cris de révolte humaine - à travers les yeux des enfants d'une classe. Comme le confie Yoshi Oïda dans le programme distribué à l'Opéra de Lyon, les enfants montrent ainsi « leur profonde distance à l'égard des conflits adultes ». Il ajoute : " Je souhaite qu'ils apparaissent comme les spectateurs d'une pièce à laquelle ils sont étrangers. Une sorte de troisième espace entre la scène et la salle, en somme. Les enfants regardent les conflits des adultes avec leurs propres préoccupations. Le public voit le spectacle montré aux enfants ; il est alors libre de rentrer dedans ou de rester à l'extérieur ». C'est cependant oublier que Yoshi Oïda a choisi spécifiquement les yeux des enfants d'une école chrétienne, conduite ici par une religieuse (sur la scène, Karine Locatelli, qui dirige la maîtrise de l'Opéra de Lyon). Si le choix de l'école chrétienne était sans doute nécessaire parce que le chœur des enfants (Boy's choir à l'origine) chante des textes de la liturgie chrétienne, l'argument de la neutralité du regard même s'il s'agit d'enfants - s'effondre quelque peu.

10 En outre, le choix de placer les enfants sur scène déroge à une autre caractéristique très forte du War Requiem de Britten: sa spatialisation. Dans la cathédrale de Coventry, Britten avait prévu de placer les deux solistes et l'orchestre de chambre tout près du chef d'orchestre, devant lui, laissant à l'arrière-plan l'énorme masse du grand orchestre et du chœur mixte. Mais surtout, le chœur de garçons devait prendre place à distance, au fond de la cathédrale, accompagné par l'orgue. Cette spatialisation mettait en valeur les trois espaces symboliques évoqués précédemment, tout en en soulignant certaines valeurs. En effet, dans le texte d'Owen et la mise en musique de Britten, la parole chrétienne empreinte de sérénité et son rite sont mis à mal : «Plus de singeries pour eux [pour les morts], de prières ni de cloches »; « Quels cierges portera-t-on pour leur dernier voyage » ; « Au pied du Golgotha passe plus d'un prêtre ; Sur leur face il y a l'orgueil/D'une chair marquée par la Bête/Qui nie le gentil Christ»(la séquence 
détournée du Sacrifice d'Isaac étant particulièrement emblématique). De même, les paroles liturgiques sont ainsi émaillées dès le début du War Requiem d'une sonnerie de triton (do-fa\#), intervalle symbolisant autrefois le diable et le maléfice, teintant ici le texte religieux de suspicion et de doute. Quant au chœur de garçons, dont la pureté contraste subitement avec l'évocation d'atrocités (après la scène du Sacrifice), qui voudrait encore y croire ? Britten avait reçu pendant son enfance une éducation très chrétienne, mais il fut sensible à certains courants anglicans qui avaient fait scandale dans les années 60 (dont celle du "Dieu sans Dieu» de Robinson, en 1963). Les tranchées et l'absurdité de la Première Guerre mondiale avaient conduit Wilfred Owen aux mêmes doutes. D'après les choix musicaux effectués pour le War Requiem, il est clair que Britten ne croit plus à la Promesse de Jérusalem. Ainsi que le souligne Nathalie Vincent-Arnaud (2005 : 201-217), les procédés de scénarisation internes au War Requiem permettent à travers une représentation pourtant non scénique d'extirper du texte de l'œuvre les pensées de Britten, que l'on peut supposer très ressemblantes à celles d'Owen. Les trois espaces figurent trois camps: «celui des "initiés" porteurs d'un discours de désespoir et de révolte, celui de la masse des fidèles ignorants des réalités sordides de la guerre et poursuivant leur itinéraire ritualisé dans une obstination aveugle, et celui d'une enfance dont le chant de louanges divines, objet d'un traitement parfois férocement sarcastique, devient un ingrédient non négligeable d'ironie dramatique puisque c'est à eux que les soldats présentent de manière récurrente le miroir de leur destinée, d'une déchéance programmée par l'Histoire » (Vincent-Arnaud 208). Si l'ironie de l'absurdité est bien présente dans la mise en scène de Yoshi Ö̈da, son choix d'incarner le chœur d'enfants - et le fait de le basculer dans l'entière réalité met de côté la critique souterraine que Britten réalise envers la religion à travers le War Requiem.

11 Néanmoins, l'humanisme que Britten déploie dans ses œuvres et ses écrits rejoint l'humanisme de Yoshi Oïda qui transparaît dans sa mise en scène : " Sur la terre, il y a toujours un combat. Cela peut sembler humain, mais c'est le contraire du cœur. Je voudrais au contraire montrer la beauté et le mystère de l'humanité. Je voudrais, malgré l'absence constante de tranquillité dans notre société d'aujourd'hui, chercher, comme Owen a voulu le faire, la manière de trouver une paix intérieure. » Sur la scène du War Requiem de l'Opéra de Lyon, le sentiment de paix pourrait envahir la toute dernière scène: "Que les Anges te conduisent au Paradis ». C'est oublier que les cloches continuent de faire résonner leurs tritons, semant le doute. Quid de la promesse pacifique ? CEuvre de paix ou de guerre ? Xavier de Gaulle écrit ainsi : «Ce qui domine chez Britten, selon Xavier de Gaulle, c'est la sensation de "peur au ventre" » (De Gaulle 372). Philipp Rupprecht évoque quant à lui une œuvre apocalyptique ou prophétique, qui témoignerait d'une grande douleur face à l'impossibilité de paix, où même le discours religieux est impuissant. Pour réconcilier les peuples, Yoshi Oïda choisit d'utiliser les drapeaux de différentes nationalités meurtries par les combats, devenant des suaires avec lesquels la veuve enveloppera les bébés morts.

Le message pacifique de Yoshi Oïda s'inscrit dans la sobriété : " Je cherche quelque chose de très épuré, sans clichés. Au lieu de donner beaucoup d'informations, j'en donne peu... c'est au public d'interpréter ». C'est là que réside la puissance de cette mise en scène du War Requiem. Certes il y a des morts, mais ils sont toujours dissimulés dans des linceuls ou des habits, et surtout il n'y a pas de combat, pas de sang, hormis dans une scène éminemment symbolique : le Libera me, déjà mentionné. 
La puissance visuelle est portée par celle, sonore, de l'orchestre, que Britten a voulu dense, massive, mais aussi à dimension variable (avec la présence de l'orchestre de chambre sur la scène à l'Opéra, côté jardin). Subtilités de couleurs et force dramatique se succèdent sous la baguette de Daniele Rustioni, nouveau directeur musical de l'Opéra de Lyon. Le chœur, préparé par Geneviève Ellis, fait montre d'une précision et d'une intensité constantes à l'Opéra de Lyon, tout comme la Maîtrise de Lyon (dirigée par Karine Locatelli) - par ailleurs menacée de fermer, ce que nous ne pouvons que déplorer. Les trois solistes Ekaterina Scherbachenko, Lauri Vasar et le ténor Paul Groves - remarquable par ses nuances de timbre, notamment dans l'aigu - apportent par leur interprétation une humanité supplémentaire à cette composition que le musicologue Gilles Couderc nommait le " $11^{\mathrm{e}}$ opéra » de Britten. Nul doute que cette œuvre portée à la scène d'opéra par Yoshi Oïda et servie par une splendide interprétation instrumentale et vocale n'a pu que révéler avec simplicité et spontanéité sa beauté. Le metteur en scène nous confiait ainsi : "Il y a beaucoup de spectacles intéressants appréciables avec le cerveau. Moi je préfère agir avec le cœur. »

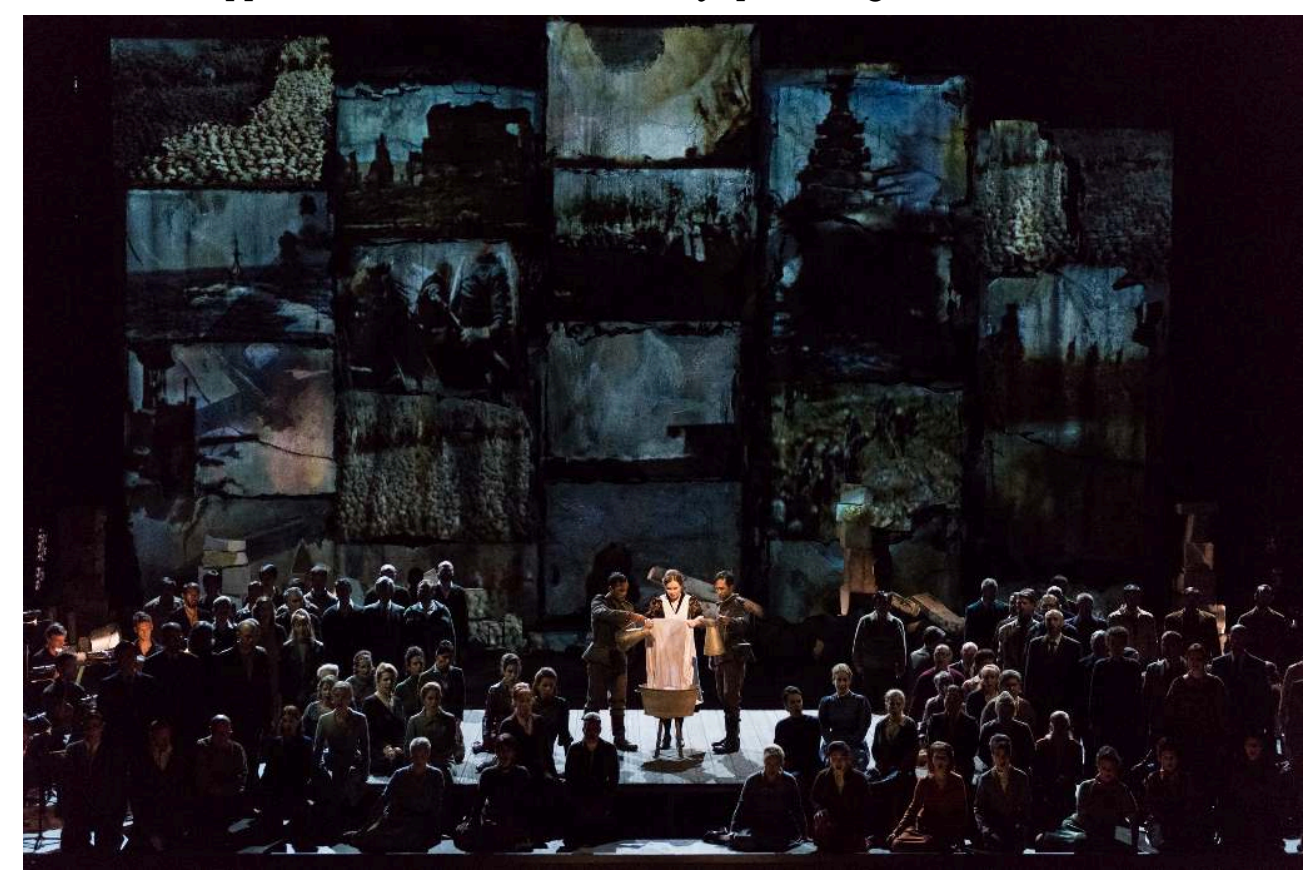




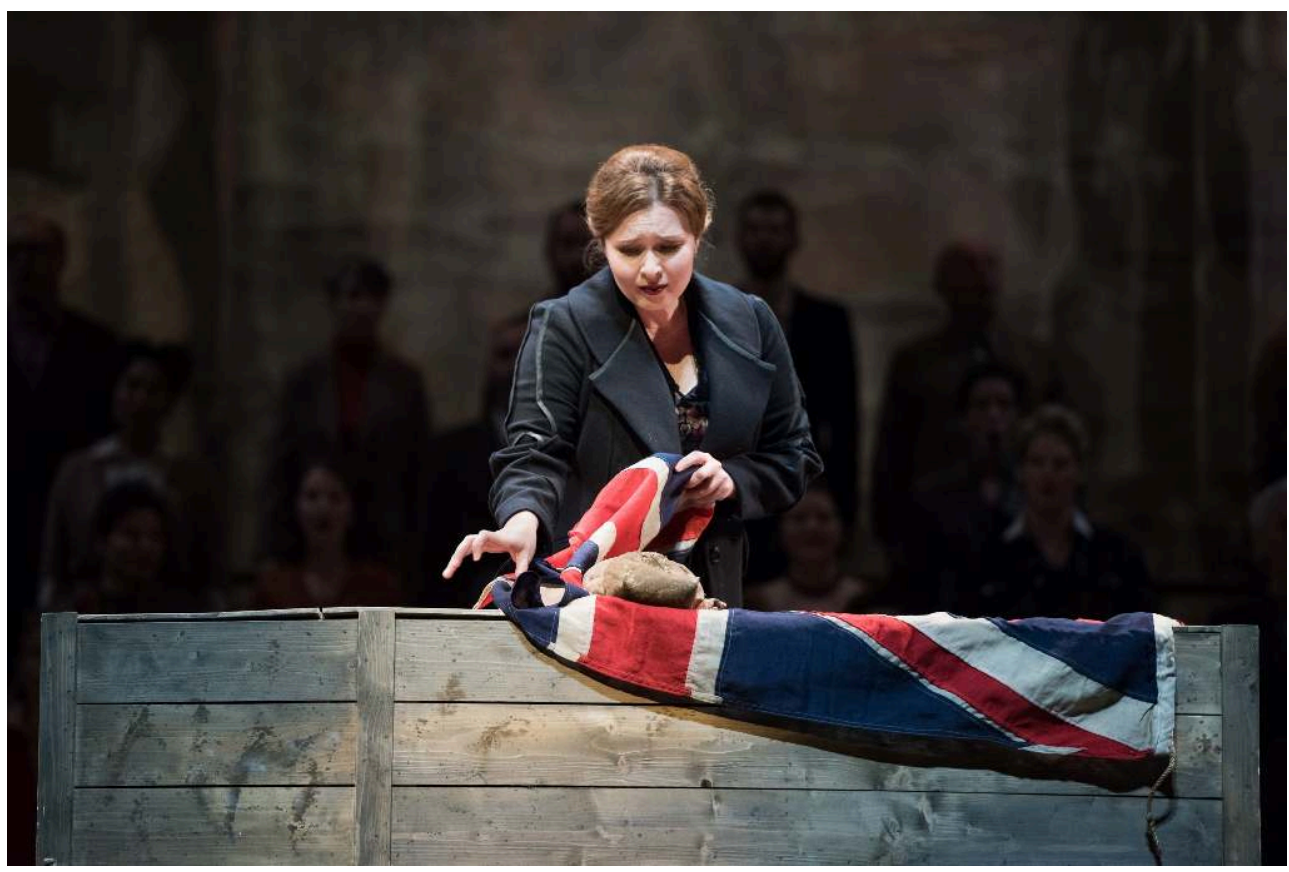

(c) Stofleth

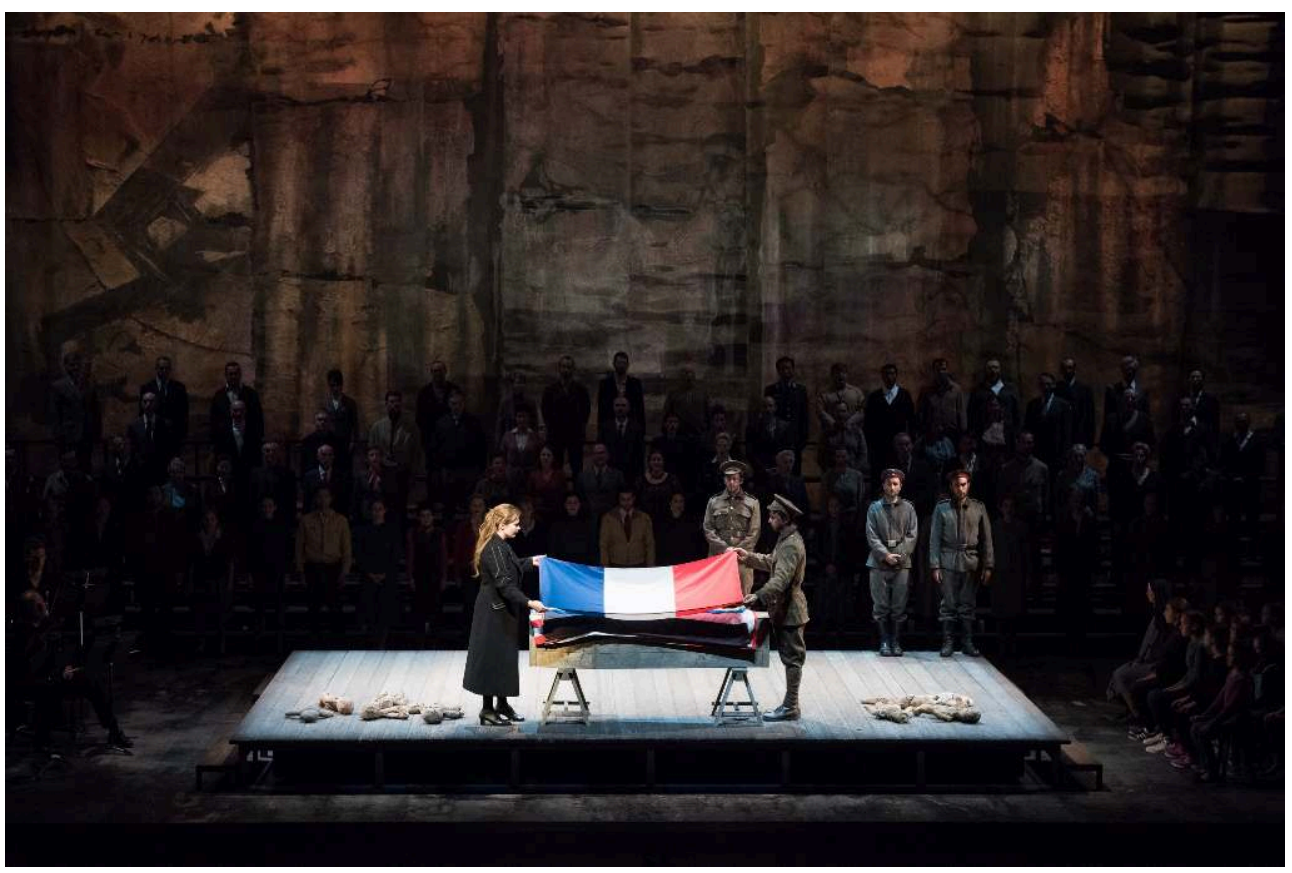

(c) Stofleth 


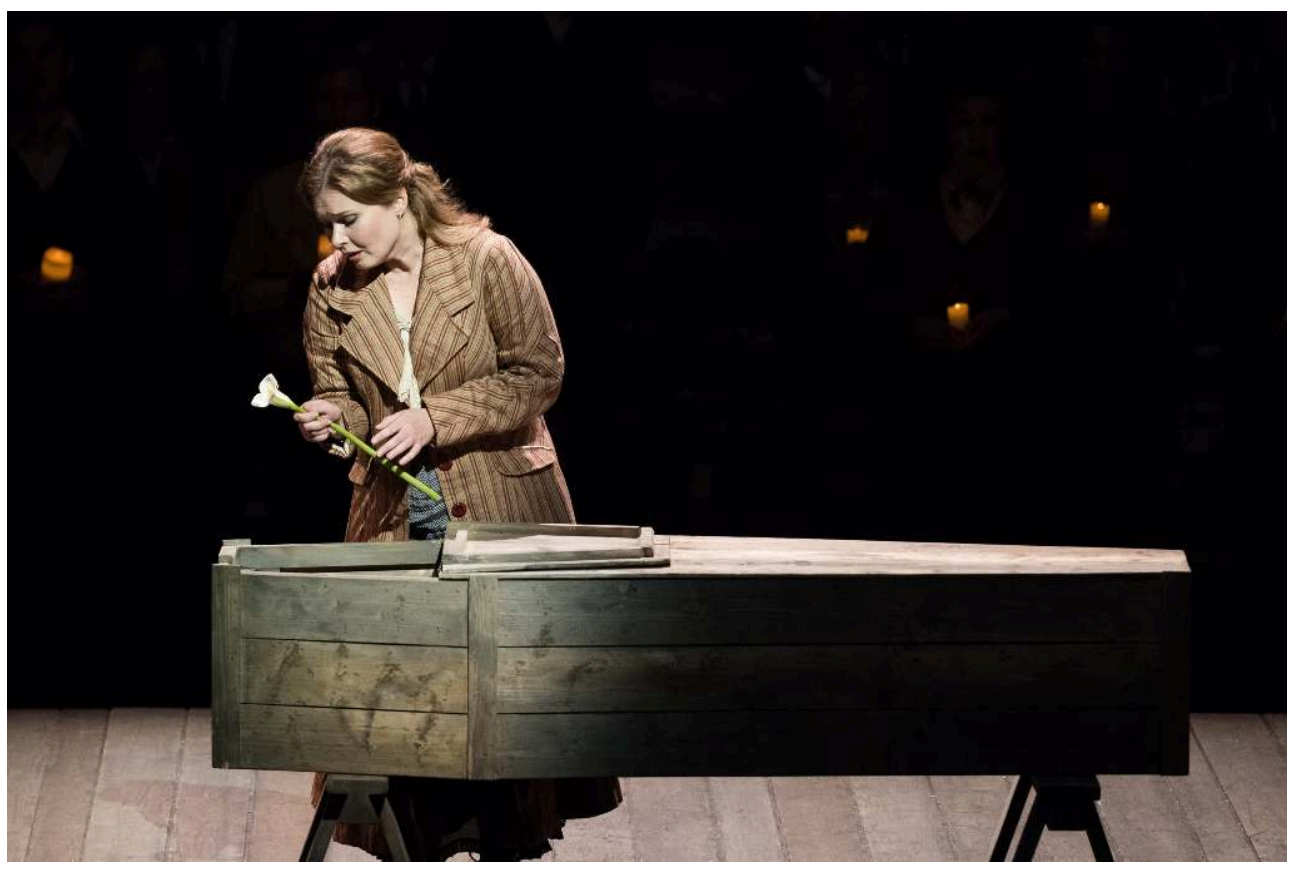

(c) Stofleth

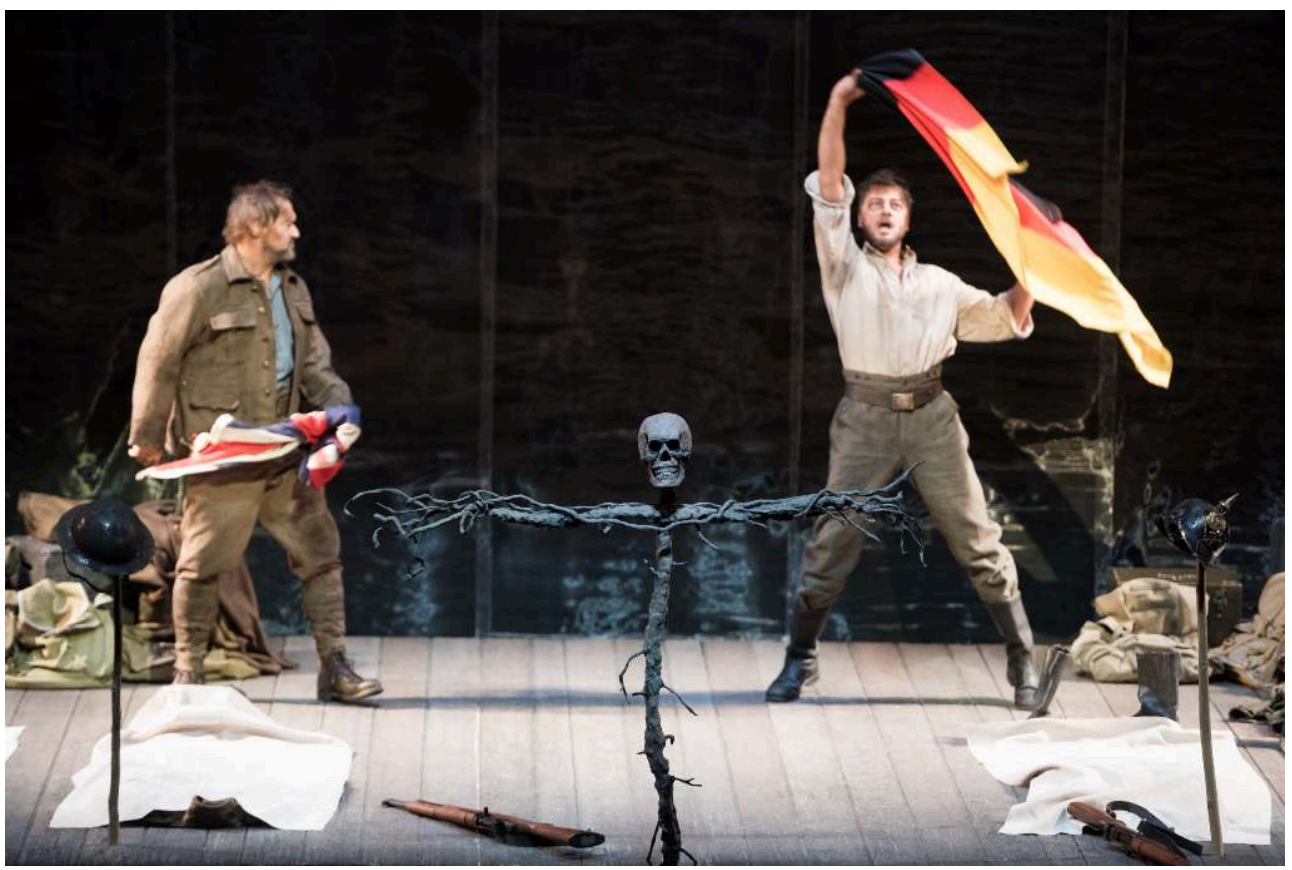

(c) Stofleth 


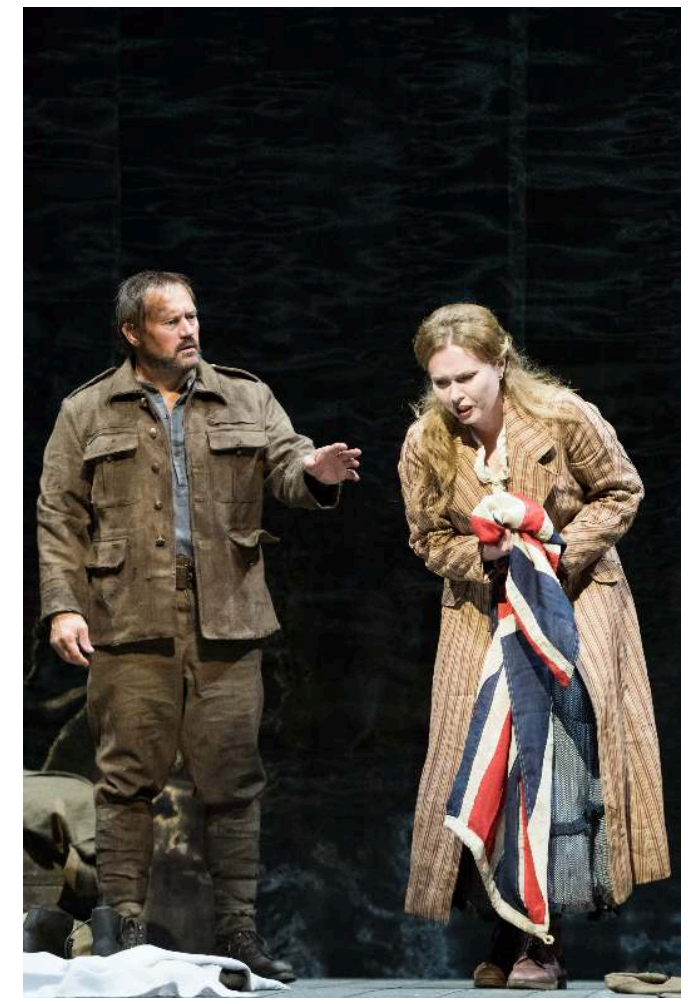

(c) Stofleth

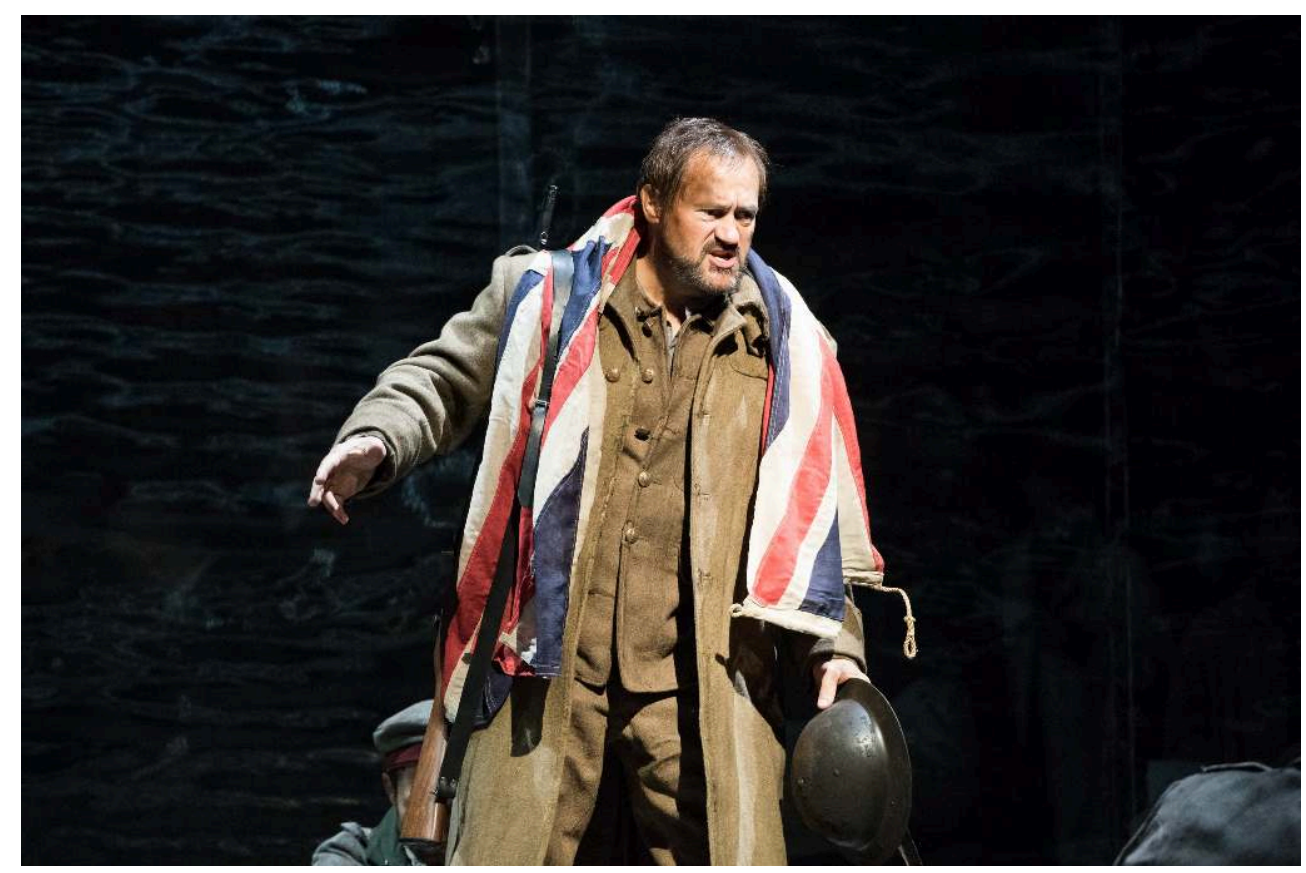

(C) Stofleth 


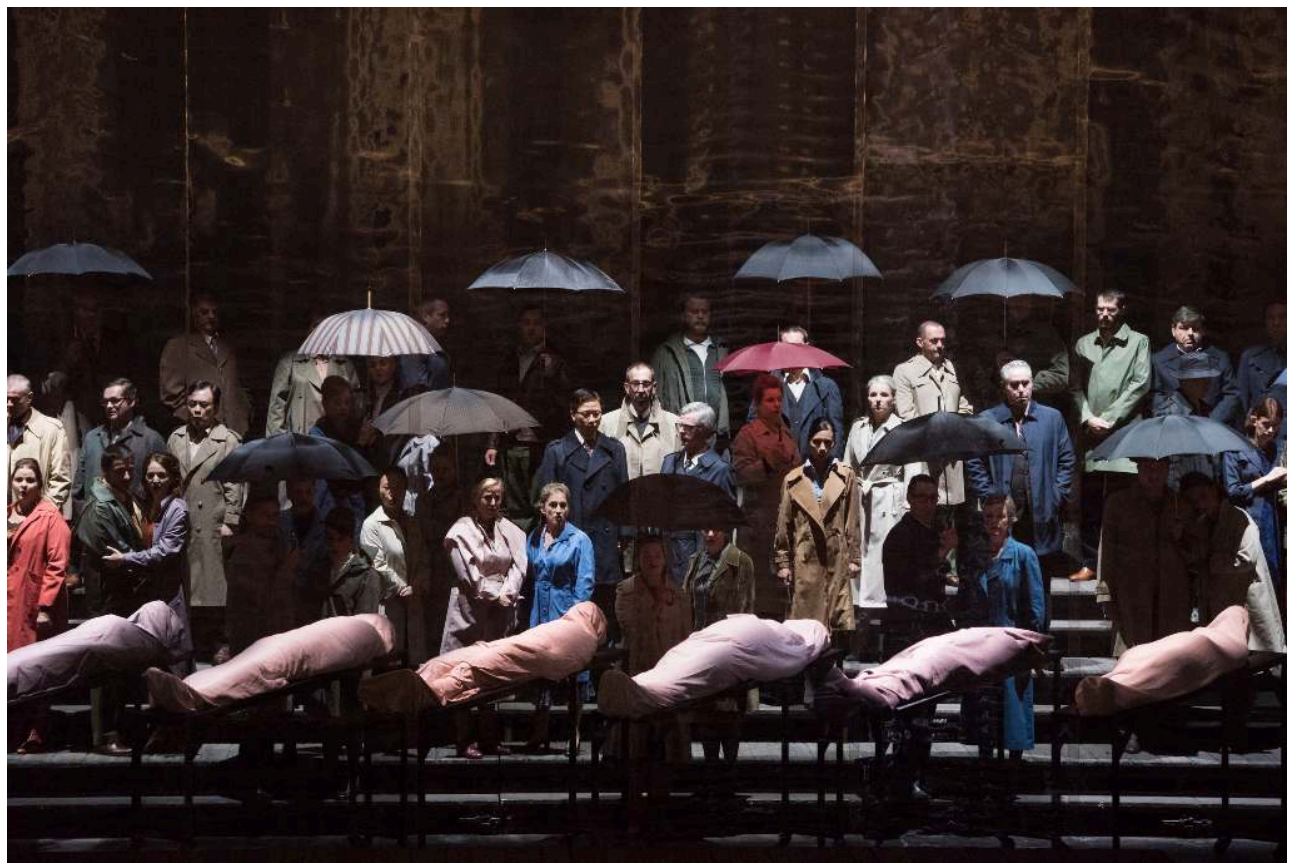

(c) Stofleth

\section{BIBLIOGRAPHIE}

Couderc, Gilles. « The War Requiem : Britten's Wilfred Owen opera ». http://www.awpreview.univparis-diderot.fr/IMG/pdf/1.1_2_coudercrevised1novbrittenwar.pdf

De Gaulle, Xavier. Benjamin Britten ou l'impossible quiétude. Arles : Actes Sud, 1996.

Rupprecht, Philippe. The Language of Benjamin Britten. Cambridge : Cambridge University Press, 2001.

Vincent-Arnaud, Nathalie. « Le dialogue et son double : aspects de la dramatisation des poèmes de Wilfrid Owen dans le War Requiem de Benjamin Britten ", in Acte(s) du colloque international " Musique et théâtralité dans les îles britanniques » (19-20 mars 2004). Metz : Centre d'Étude des Textes et Traductions, Université Paul-Verlaine, 2005 : 201-217.

\section{NOTES}

1. Sauf mention contraire, toutes les citations de Yoshi Oïda sont extraites de l'entretien que le metteur en scène a eu la gentillesse de nous accorder le vendredi 6 octobre 2017. 
INDEX

Thèmes : Music, Dance

Mots-clés : Benjamin Britten, pacifisme, Yoshi Oïda, War Requiem, Wilfred Owen

Keywords : Benjamin Britten, pacifism, Yoshi Ö̈da, War Requiem, Wilfred Owen

\section{AUTEURS}

\section{MURIEL JOUBERT}

Université Lumière-Lyon 2

Muriel.Joubert@univ-lyon2.fr 